\title{
Study of Meta-analysis strategies for network inference using information-theoretic approaches
}

\author{
Ngoc C. Pham ${ }^{1 *}$ (D), Benjamin Haibe-Kains $2,3,4,5$, Pau Bellot ${ }^{6}$, Gianluca Bontempi ${ }^{7}$ and Patrick E. Meyer ${ }^{1}$
}

\author{
${ }^{*}$ Correspondence: \\ camngoc.pham@doct.ulg.ac.be \\ 1 Bioinformatics and Systems \\ Biology (BioSys) Lab, Université de \\ Liège, Liège, Belgium \\ Full list of author information is \\ available at the end of the article
}

\begin{abstract}
Background: Reverse engineeringof gene regulatory networks (GRNs) from gene expression data is a classical challenge in systems biology. Thanks to high-throughput technologies, a massive amount of gene-expression data has been accumulated in the public repositories. Modelling GRNs from multiple experiments (also called integrative analysis) has; therefore, naturally become a standard procedure in modern

computational biology. Indeed, such analysis is usually more robust than the traditional approaches, which suffer from experimental biases and the low number of samples by analysing individual datasets.

To date, there are mainly two strategies for the problem of interest: the first one ("data merging") merges all datasets together and then infers a GRN whereas the other ("networks ensemble") infers GRNs from every dataset separately and then aggregates them using some ensemble rules (such as ranksum or weightsum). Unfortunately, a thorough comparison of these two approaches is lacking.
\end{abstract}

Results: In this work, we are going to present another meta-analysis approach for inferring GRNs from multiple studies. Our proposed meta-analysis approach, adapted to methods based on pairwise measures such as correlation or mutual information, consists of two steps: aggregating matrices of the pairwise measures from every dataset followed by extracting the network from the meta-matrix. Afterwards, we evaluate the performance of the two commonly used approaches mentioned above and our presented approach with a systematic set of experiments based on in silico benchmarks.

Conclusions: We proposed a first systematic evaluation of different strategies for reverse engineering GRNs from multiple datasets. Experiment results strongly suggest that assembling matrices of pairwise dependencies is a better strategy for network inference than the two commonly used ones.

Keywords: Meta-analysis, Gene regulatory networks, Systems biology, Gene expression, Mutual information 


\section{Background}

One of the most long-standing challenges in Systems Biology is the development of methods, which are able to construct the complete set of regulatory interactions of a cell. The regulating circuitry, also called gene regulatory network (GRN), can then be used by bio-medical experts to understand key mechanisms in cells. Thanks to high-throughput technologies, a large amount of transcriptome data is now available through public repositories (e.g. NCBI GEO [1], ArrayExpress [2]), providing opportunities to study the GRNs of many organisms.

In the last decade, a variety of algorithms have been proposed in an attempt to address this reverse engineering problem. These algorithms can be classified into several categories [3], such as: regression-based, pairwise similarity (mutual information, correlation,...), Bayesian networks or even ensemble approaches (that combine several different approaches). Among those, mutual information (MI) based algorithms, such as CLR [4], ARACNE [5], MRNET [6, 7] and so on, gather more and more attention owing to their capability to deal with up to several thousands of variables in the presence of a limited number of samples [7]. Generally, MI-based algorithms start by estimating a pairwise mutual information (i.e. a non-linear dependency measure) between all pairs of genes, resulting in a mutual information matrix (MIM). Afterwards, indirect interactions are eliminated from the MIM by the different approaches and subsequently a GRN is inferred.

Since a single dataset has typically a small sample size (usually less than 200 observations) and suffers from potential experimental biases, classical reverse engineering algorithms, which relies only on a standalone dataset, show their limits in unravelling reliably underlying interactions. By contrast, integrative analysis of multiple studies is able to increase significantly the statistical power and thus is becoming a standard procedure in modern computational biology [8]. Nevertheless, the question of how to integrate data consistently and efficiently raises new challenges [9].

In the mean time, meta-analysis strategies have been increasingly used for detecting differentially expressed genes from microarray data [10]. In the meta-analysis approaches, each single dataset is analysed separately and then the final results are combined [11]. Several strategies have been proposed in order to perform meta-analysis on expression data. For instance, a meta-analysis of public gene expression data and clinical data was conducted by using the concept of "coexpression" modules to reveal various results of previous gene expression studies in breast cancer [12, 13]. In another research [14], Hong et al. developed a Bioconductor package RankProd that allow researchers to do metaanalysis under two experimental microarray conditions to identify differentially expressed genes.

While the problem of detecting differentially expressed genes across several studies has been intensively studied, it is, however, not yet the case when it comes to constructing GRNs.

To deal with the challenge of meta-network inference, there have been plenty of proposed methods, which can be divided into two main categories: "data merging" and "network ensemble". In the "data merging" approach, datasets are integrated at the expression level into a unique dataset, from which GRNs are inferred [15-17]. However, one of the major problem of this approach is the removal of batch effects. Indeed, the use of different platforms, and different methodologies by different research groups introduce statistical biases (batch effects) that can lead to incorrect conclusions [18]. For example, 
it is known that normalization techniques, such as RMA [19], consisting in re-scaling gene expression values at the probe intensity level for Affymetrix data [20], is not able to remove batch effects. Consequently, batch removal methods, like COMBAT [21], is typically used before merging data [18].

On the other hand, "ensemble" methods of merging GRNs from different datasets, i.e. by weighting gene-gene interactions according to their average rank in each network [3], have emerged as an alternative to the "data merging" approach. This approach rooted in the "wisdoms of crowds" concept, which was first introduced in the DREAM5 challenge and then further developed by [22] with the TopkNet algorithms to produce consensus networks.

In this paper, we also introduce a new meta-analysis strategy to build consensus networks. The new strategy consists in aggregating matrices of pairwise mutual information with each being estimated from a gene expression dataset to produce a meta-matrix, from which a GRN is inferred using classical information-theoretic network inference algorithms. Additionally, the paper presents the first thorough experimental comparison of these three "meta" approaches for the reconstruction of networks, namely "data merging", "network ensemble" and "coexpression matrices aggregation". The performances of these three sets of methods are evaluated using synthetic datasets from the standard Bioconductor netbenchmark package.

\section{Methods}

State-of-the-art

Mutual information is a non-linear measure of dependency between two variables (genes) $X$ and $Y$, defined as follow

$$
I(X, Y)=\sum_{x \subset X, y \subset Y} p(x, y) \log \frac{p(x, y)}{p(x) p(y)}
$$

where $p(x, y)$ is the joint probability distribution of $X$ and $Y$, and $p(x)$ and $p(y)$ are the marginal probability distributions of $X$ and $Y$, respectively.

This dependency measure has been used for reconstructing networks by several methods such as CLR, ARACNE or MRNET. The first one - CLR method (The Context Likelihood or Relatedness network) [4] creates an edge between each pair of genes $i$ and $j$ if the combined $z$-score of the mutual information between them is above a given threshold, where the combined $\mathrm{z}$-score is defined as:

$$
c_{i j}=\sqrt{c_{i}^{2}+c_{j}^{2}} \text { with } c_{i}=\max \left(0, \frac{M_{i j}-\mu_{M_{i}}}{\sigma_{M_{i}}}\right)
$$

in which, $\mu_{M_{i}}$ and $\sigma_{M_{i}}$ are the mean and standard deviation of the empirical distribution of the mutual information of gene $i$.

The second algorithm named ARACNE (The Algorithm for the Reconstruction of Accurate Cellular Networks) [5] relies on the "Data Processing Inequality" (DPI) which removes the edge with the weakest mutual information, in every triplet of genes.

And finally, the Minimum Redundancy NETworks (MRNET) [6, 7] method reconstructs a network using the feature selection technique known as Minimum Redundancy Maximum Relevance (MRMR) [23]. The minimum redundancy criterion makes the 
implicit assumption that variables with redundant information to the most relevant variables are indirect links.

Using these three information-theoretic network inference techniques, which are available from the Bioconductor Minet package, we will evaluate the performance of the three meta-analysis approaches that were demonstrated in Fig. 1 in the next sections.

\section{Data merging - D methods}

A straightforward approach for performing integrative analysis of multiple studies is combining all datasets together and then analysing the merged dataset. These method, named "data merging" and denoted here with the letter (D), were widely used in [15-17] to reconstruct large-scale GRNs because of their simplicity. However, since high dimensional data often suffers from unwanted biases, a variety of techniques can be used to correct for

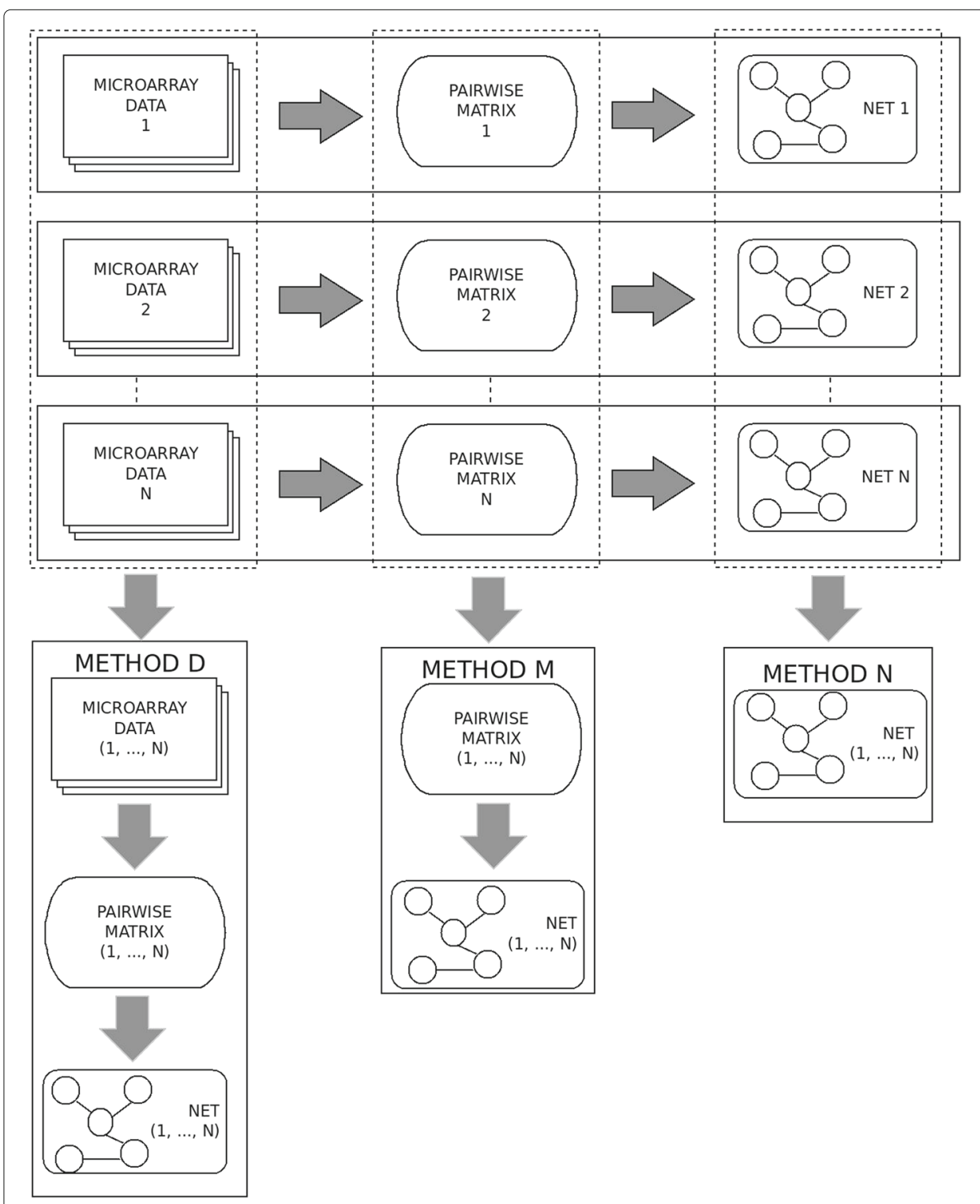

Fig. 1 Meta-network strategies: assembling datasets, pairwise matrices or networks 
these non-biological variations. We present in the following two classical scaling methods typically used to assemble datasets, and one batch-effect-removal method.

Normalization: $B M C(D 1)$ and z-score (D2)

Let $X$ be a matrix $X^{m \times n}$ denoting the dataset of gene expression values. In this matrix, columns represent samples and rows represent genes, and $x_{i j}$ represents the expression value of gene $i$ in sample $j$ of dataset $X$. In [24], a normalization technique named BMC (Eq. 3) was applied for merging breast cancer datasets.

$$
\hat{x_{i j}}=x_{i j}-\bar{x}_{i}
$$

Similarly, the z-score normalization [25] is described by Eq. 4 and was also included for evaluation.

$$
\hat{x_{i j}}=\frac{x_{i j}-\bar{x}_{i}}{\sigma_{x_{i}}}
$$

\section{Batch effects removal: COMBAT(D3)}

Gene expression datasets mostly come from different platforms and laboratories, causing the so-called batch effects. Consequently, batch removal methods, like COMBAT (also known as Empirical Bayes) [21], is often used to detect and remove this inevitable variation. COMBAT, which was shown to outperform other commonly used batch removal methods in some specific scenarios [26], uses estimations for the LS (location-scale) parameters (e.x. mean and variance) for each gene independently [27]. The gene, afterwards, is adjusted to meet the estimated model. In this paper, combining datasets using the COMBAT algorithm will be included for comparison and referred as method D3.

\section{Networks ensemble - $\mathrm{N}$ methods}

As we presented in the previous subsection, one of the difficulties of the data-merging methods is how to handle the batch effects. Consequently, "networks ensemble" method (denoted with the letter $(\mathrm{N})$ in the paper) has been proposed as an alternative approach. In fact, by combining topologies of networks rather than datasets we are able to avoid dealing with batch effects. This method first constructs every single transcriptional networks independently before combining them to produce a so-called community network [3]. In general, combining networks consists in two distinct steps: transformation and aggregation [28]. Indeed, before assembling networks, a network-normalization step can be performed because it is common to observe networks that exhibit different distribution of edge weights.

Let $e_{i j}$ be the weight of an edge between gene $i$ and gene $j$ and $t_{n}\left(e_{i j}\right)$ be the normalized value for $e_{i j}$ in the network $n$. In the next subsections, we discuss three viable combinations of network transformation and aggregation.

\section{RankSum method (N1)}

The RanSum method, which was introduced in [3], is based on rank averaging: If $e_{i j}$ denotes an edge connecting genes $i$ and $j$ and $r_{n}\left(e_{i j}\right)$ the rank of the edge in network $n$, the final rank of the edge across $N$ networks is computed by:

$$
r\left(e_{i j}\right)=\sum_{n=1}^{N} r_{n}\left(e_{i j}\right)
$$


Internal quality control index (N2)

In [10], six quantitative quality control measures have been proposed for the inclusion/exclusion of gene expression studies used for the meta-analysis. Among these measures, the internal quality control index will be included in this paper, as method N2 for assembling networks. Let the similarity between two studies $m$ and $n$ be defined as

$$
r_{m n}=\operatorname{spcor}\left(\left(t_{n}\left(e_{i j}\right) ; 1 \leq i \leq j \leq G\right),\left(t_{m}\left(e_{i j}\right) ; 1 \leq i \leq j \leq G\right)\right)
$$

In which $r_{m n}$ is the Spearman's rank correlation of the pairwise correlation structure between study $m$ and $n$ and $G$ represents the total number of genes in the studies. The dissimilarity (or distance) between study $m$ and $n$ is defined as $d_{m n}=\left(1-r_{m n}\right) / 2$. For a given study $k$, a weight $-w_{k}$ will be granted as the fraction between the sum of distances between study $k-D_{k}^{*}$ to all other studies and the sum of pairwise distances between all studies excluding the study $k-D_{k}^{\#}$ with

$$
D_{k}^{*}=\left\{d_{k n}\right\}_{1 \leq n \leq N ; n \neq k} \text { and } D_{k}^{\#}=\left\{d_{m n}\right\}_{1 \leq m \neq n \leq N ; m \neq k ; n \neq k}
$$

Afterwards, the weight of the edge between two variables (genes) $X$ and $Y$ is aggregated by the following equation:

$$
\hat{e}_{I Q C}(X ; Y)=\frac{\sum_{k=1}^{N} w_{k} t_{k}\left(e_{X Y}\right)}{\sum_{k=1}^{N} w_{k}}
$$

\section{Median method (N3)}

In [22] the median value was introduced for aggregating consensus networks. This method assigns the median value among $N$ values representing the confidence score of a specific edge in $N$ different networks.

$$
a_{M}\left(e_{i j}\right)=\operatorname{median}\left\{t_{1}\left(e_{i j}\right), \ldots, t_{N}\left(e_{i j}\right)\right\}
$$

\section{Matrices of coexpression based aggregation approaches - $M$ methods}

Our new category of meta-analysis approaches (denoted with the letter $(M)$ in this paper) aggregates mutual information matrices rather than data or networks. The idea behind assembling pairwise matrices is that, although expression data typically shows high variability due to differences in technology, samples, labels, etc., pairwise dependency measures between genes should be much less variant (i.e. dependent variables, such as a regulating variable and its regulated counterpart, should remain dependent in every platform/experiment/dataset even if ranges of values differ greatly). Thus, to infer a network from various expression data, our approach consists in combining mutual information matrices (MIMs) estimated independently from each dataset. Then a GRN network is inferred from the aggregated MIMs. In the following subsections, we will demonstrate three feasible methods to assemble matrices of pairwise measure.

\section{Random-effects model (M1)}

It should be noted that the problem of combining MIMs across multiple datasets can be framed in the context of a meta-analysis of correlation coefficients [29]. Hunter and 
Schmidt [30] introduced a single random-effects method based on untransformed correlation coefficients, at which datasets are weighted simply by the sample sizes on which each effect size (the estimated MIM) is based. Our first weighting schema (method M1), described by Eq. 10, utilises this random-effects method, but using MI instead of correlations.

$$
\hat{I}_{R E}(X ; Y)=\frac{\sum_{k=1}^{N} n_{k} I\left(X_{k} ; Y_{k}\right)}{\sum_{k=1}^{N} n_{k}}
$$

where $I\left(X_{k} ; Y_{k}\right)$ is the MI between two variable $X_{k}$ and $Y_{k}$ in the study $k$ and $n_{k}$ is the number of samples of study $k$.

The idea is simply that effect sizes based on large samples will be more precise than those based on small samples.

\section{Internal quality control index (M2)}

Here, the internal quality control index measure was used again with some minor modifications. First, the similarity between two studies $m$ and $n$ was defined as

$$
r_{m n}=\operatorname{spcor}\left(\left(I_{m i j} ; 1 \leq i \leq j \leq G\right),\left(I_{n i j} ; 1 \leq i \leq j \leq G\right)\right)
$$

Then, the MI between two variables (genes) $X$ and $Y$ is aggregated by the following equation:

$$
\hat{I}_{I Q C}(X ; Y)=\frac{\sum_{k=1}^{N} w_{k} I\left(X_{k} ; Y_{k}\right)}{\sum_{k=1}^{N} w_{k}}
$$

\section{Median method (M3)}

One of the major issue of M1 is that the quality of datasets used in meta-analysis is not explicitly taken into account. Indeed, inclusion of poor quality datasets is likely to weaken statistical power [10]. Thus, an alternative schema for combining MIMs across heterogeneous studies namely method M3 can be proposed. Method M3 is explained by formula 13, in which the aggregated MI of a gene pair $X$ and $Y$ is the median value of all MI values between them across all studies.

$$
\hat{I}_{M}(X, Y)=\operatorname{median}\left(I\left(X_{1}, Y_{1}\right), I\left(X_{2}, Y_{2}\right), \ldots I\left(X_{N}, Y_{N}\right)\right)
$$

\section{Results}

\section{Simulated datasets}

There are two tasks one needs to consider in order to validate networks: 1) defining a "gold standard" - which is a set of true regulations describing the underlying interaction network, 2) selecting quantitative measures to statistically assess the quality of inferred networks. Typically, the first task is addressed by collecting well-known regulations mined from literature with strong supporting evidences. However, those regulations just cover a small part of the underlying network and therefore cannot be an ideal reference network to thoroughly compare methods. Hence the latter approach is often completed by in-silico experiments. 
In this paper, in silico benchmarks are selected from every one of the 4 biological networks and artificially generated datasets coming from the Netbenchmark Bioconductor package [31]. The selected datasets are generated by two simulators namely GNW and SynTReN. The GNW simulator generates network structures by extracting parts of known real GRN structures capturing several of their important structural properties while the SynTReN simulator generates the underlying networks by selecting sub-networks from E. coli and Yeast organisms [31]. The characteristics of the 4 biological networks are presented in more detail in Table 1.

In the following step, each large dataset will be split into 6 sub-datasets with a number of experiments ranging between 30 to 300 (a number chosen randomly in order to simulate real case scenario where there is a variety of the number of samples). For example, in Fig. 2, an original dataset is split into 6 sub-datasets with the following number of samples: 50, 100, 150, 120, 70 and 190. Additionally, two extremely noisy studies are added, both with a large sample size for each (between 280 and 300). Those datasets allow to test the sensitivity of meta-network methods to datasets that should typically be excluded. Indeed, a few biological studies dating back to the beginning of the microarray technology have very little information and are typically excluded from meta-analysis studies.

\section{Network prediction and validation for simulated datasets}

In order to make the network inference problem more challenging and realistic, noise and transformations of data are added. In particular, we define three levels of data-distortion:

i) Level 1: An independent lognormal noise call "global" noise, with intensity between 20 and $50 \%$, is added to the first 6 datasets. The standard deviation of this noise $\left(\sigma_{\text {Global }}\right)$ is the same for the whole dataset and is a percentage $\left(\kappa_{g} \%\right)$ of the mean variance of all the genes in the $\operatorname{dataset}\left(\bar{\sigma}_{g}\right)$. It is defined as follows:

$\sigma_{\text {Global; } \kappa_{g} \%}=\bar{\sigma}_{g} \frac{U(0.8 \kappa, 1.2 \kappa)}{100}$.

ii) Level 2: In addition to the global noise, a normally distributed "local" noise with intensity also ranging between 20 and 50\%, is added. This is an additive Gaussian noise with zero mean and a standard deviation $\left(\sigma_{\operatorname{Local}(g)}\right)$ that is around a percentage $(\kappa \%)$ of the gene standard deviation $\left(\sigma_{g}\right)$. Therefore, the Signal-to-Noise-Ratio(SNR) of each gene is similar. The local noise standard deviation can be formulated as follows: $\sigma_{\operatorname{Local}(g) ; \kappa \%}=\sigma_{g} \frac{U(0.8 \kappa, 1.2 \kappa)}{100}$ where $U(a, b)$ is a uniform distribution between $a$ and $b$.

iii) Level 3: In addition to the two previous noises, each sub-dataset can be transformed using a randomly chosen non-linear transformation such as $x^{2}$ or $\log (x)$. This random data transformation is not really meant to be realistic but rather to allow us to better assess the behaviour of each meta-method when faced with extreme distortion. It is worth emphasizing that the two non-informative studies remain unchanged across all experiments. A flowchart of this process is illustrated in Fig. 2.

Table 1 Networks used in the paper

\begin{tabular}{llllll}
\hline Network & Name & Topology & Experiments & Genes & Edges \\
\hline SynTreN $_{300}$ & S1 & E. coli & 800 & 300 & 468 \\
SynTreN $_{1000}$ & S2 & E. coli & 1000 & 1000 & 4695 \\
GNW $_{1565}$ & G1 & E. coli & 1565 & 1565 & 7264 \\
GNW $_{2000}$ & G2 & Yeast & 2000 & 2000 & 10392 \\
\hline
\end{tabular}




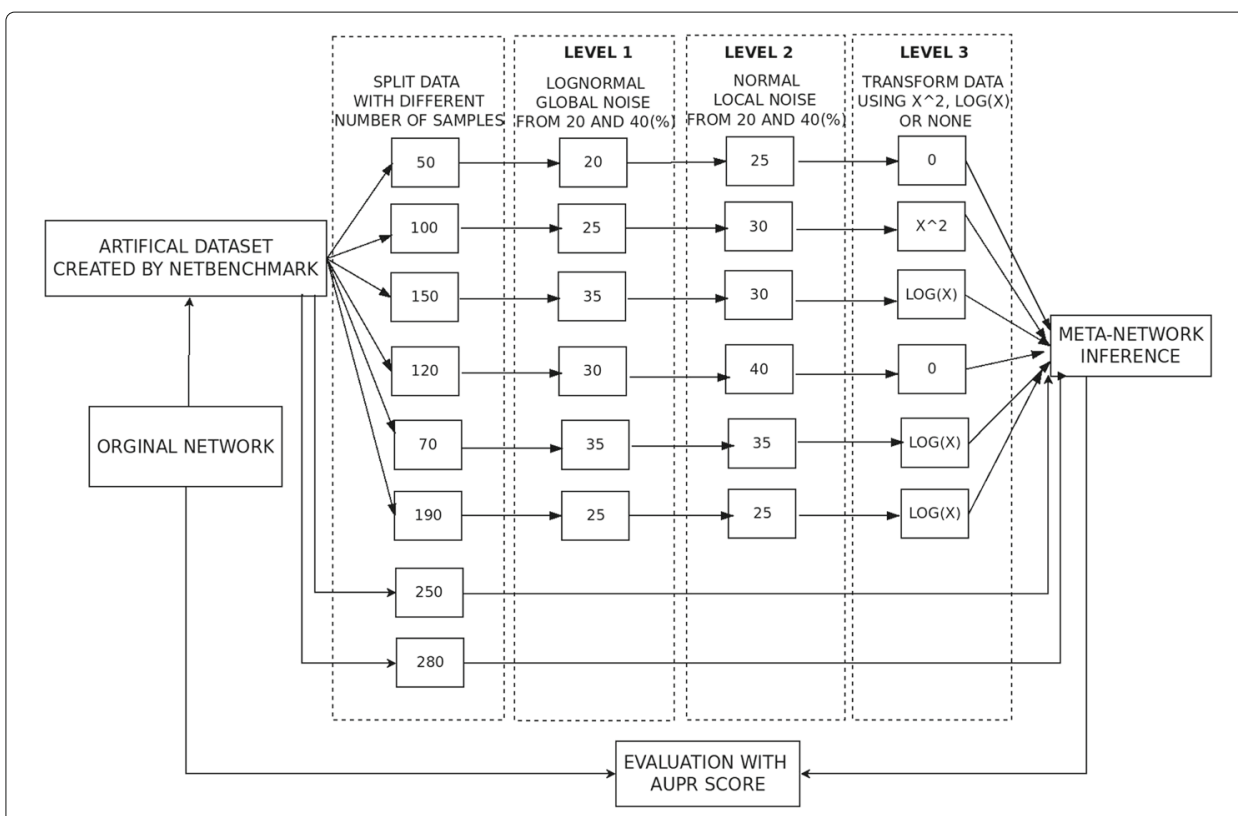

Fig. 2 Framework for data collection, network prediction and validation

The schema for network prediction and validation is also illustrated in Fig. 2. Initially, all methods (three D, M and N, totalling nine) are used to construct a consensus GRN from the split datasets. All methods are assessed on 12 challenges (three levels of distortion for four datasets). Finally, the process is repeated for the three information-theoretic inference methods, hence totalling 36 challenges. This is done to make sure that our analysis is not method specific.

Given the ground-truth knowledge of the simulated data, traditional statistical error measures, such as F-score, AUCROC (Area Under the Receiver Operating Characteristic curve) or AUPR (Area Under the Precision-Recall curve) can be used to verify the quality of networks at the global-level [32]. ROC curves, however, can present an overly optimistic view of an algorithm's performance if there is a large skew in the class distribution [33], which is generally the case in network inference because of its spareness. Consequently, PR curves, which are often used in information retrieval, have been recommended as an alternative to ROC curves [33]. The AUPR for each GRN is, thus, selected to report for all methods in each challenge of the study. Due to the randomization of various experimental parameters (noise intensity, number of samples), 10 repetitions are made. Finally, the average of the ten AUPR values, for each method on each challenge, is presented. Furthermore, in order to see how significantly better is the best method, a pvalue using a Wilcoxon test [34] and adjusted, using a Bonferroni correction [35], between each approach and the best one is computed.

\section{Experimental results}

In this section, we present the experimental results of all presented methods for reconstructing GRNs from multiple expression datasets (Table 2). For the D family of methods, it can be observed that normalization using z-score transformation (D2) is better than BMC (D1). This conclusion is true for all three network inference algorithms used in this 
Table 2 Area under PR-Curves (the higher the better) for 9 methods on 4 datasets with 3 levels of increasing data-distortion

\begin{tabular}{|c|c|c|c|c|c|c|c|c|c|c|}
\hline \multicolumn{2}{|r|}{ MRNET } & \multirow{2}{*}{$\begin{array}{l}\text { D1 } \\
0.082\end{array}$} & \multirow{2}{*}{$\frac{D 2}{0.116}$} & \multirow{2}{*}{$\frac{\text { D3 }}{0.107}$} & \multirow{2}{*}{$\frac{N 1}{0.052}$} & \multirow{2}{*}{$\frac{\mathrm{N} 2}{0.138}$} & \multirow{2}{*}{$\frac{\mathrm{N} 3}{0.124}$} & \multirow{2}{*}{$\begin{array}{l}M 1 \\
\mathbf{0 . 1 4 1}\end{array}$} & \multirow{2}{*}{$\frac{M 2}{0.137}$} & \multirow{2}{*}{$\begin{array}{l}\text { M3 } \\
0.121\end{array}$} \\
\hline S1 & Level 1 & & & & & & & & & \\
\hline & Level 2 & 0.078 & 0.110 & 0.101 & 0.051 & 0.119 & 0.116 & 0.120 & 0.117 & 0.102 \\
\hline & Level 3 & 0.088 & 0.099 & 0.096 & 0.050 & 0.116 & 0.114 & 0.120 & 0.112 & 0.105 \\
\hline \multirow[t]{3}{*}{ S2 } & Level 1 & 0.013 & 0.016 & 0.016 & 0.023 & 0.034 & 0.026 & 0.046 & 0.043 & 0.026 \\
\hline & Level 2 & 0.013 & 0.016 & 0.016 & 0.024 & 0.023 & 0.021 & 0.026 & 0.025 & 0.019 \\
\hline & Level 3 & 0.014 & 0.016 & 0.016 & 0.024 & 0.024 & 0.021 & 0.027 & 0.025 & 0.020 \\
\hline \multirow[t]{3}{*}{ G1 } & Level 1 & 0.051 & 0.099 & 0.122 & 0.051 & 0.125 & 0.129 & 0.156 & 0.142 & 0.131 \\
\hline & Level 2 & 0.037 & 0.087 & 0.108 & 0.049 & 0.108 & 0.115 & 0.138 & 0.122 & 0.116 \\
\hline & Level 3 & 0.039 & 0.077 & 0.101 & 0.048 & 0.104 & 0.113 & 0.133 & 0.115 & 0.111 \\
\hline \multirow[t]{6}{*}{ G2 } & Level 1 & 0.028 & 0.050 & 0.073 & 0.029 & 0.106 & 0.097 & 0.131 & 0.126 & 0.097 \\
\hline & Level 2 & 0.023 & 0.046 & 0.066 & 0.028 & 0.089 & 0.084 & 0.116 & 0.111 & 0.085 \\
\hline & Level 3 & 0.029 & 0.044 & 0.066 & 0.028 & 0.088 & 0.085 & 0.113 & 0.111 & 0.087 \\
\hline & Mean & 0.041 & 0.065 & 0.074 & 0.038 & 0.090 & 0.087 & 0.106 & 0.099 & 0.085 \\
\hline & o-value & .00195 & .00195 & .00195 & .00195 & .00195 & .00195 & & .00195 & .00195 \\
\hline & \multicolumn{10}{|c|}{ ARACNE } \\
\hline \multirow[t]{3}{*}{ S1 } & Level 1 & 0.032 & 0.043 & 0.042 & 0.045 & 0.101 & 0.030 & 0.063 & 0.055 & 0.051 \\
\hline & Level 2 & 0.034 & 0.042 & 0.040 & 0.036 & 0.080 & 0.022 & 0.045 & 0.046 & 0.039 \\
\hline & Level 3 & 0.038 & 0.039 & 0.038 & 0.038 & 0.083 & 0.023 & 0.049 & 0.049 & 0.047 \\
\hline \multirow[t]{3}{*}{ S2 } & Level 1 & 0.005 & 0.005 & 0.006 & 0.017 & 0.020 & 0.006 & 0.025 & 0.022 & 0.013 \\
\hline & Level 2 & 0.005 & 0.005 & 0.005 & 0.015 & 0.015 & 0.005 & 0.014 & 0.013 & 0.009 \\
\hline & Level 3 & 0.005 & 0.005 & 0.005 & 0.015 & 0.015 & 0.005 & 0.013 & 0.012 & 0.008 \\
\hline \multirow[t]{3}{*}{ G1 } & Level 1 & 0.030 & 0.061 & 0.083 & 0.126 & 0.119 & 0.075 & 0.131 & 0.116 & 0.102 \\
\hline & Level 2 & 0.022 & 0.054 & 0.071 & 0.102 & 0.092 & 0.056 & 0.105 & 0.090 & 0.087 \\
\hline & Level 3 & 0.025 & 0.047 & 0.068 & 0.105 & 0.096 & 0.058 & 0.109 & 0.096 & 0.086 \\
\hline \multirow[t]{6}{*}{ G2 } & Level 1 & 0.013 & 0.028 & 0.048 & 0.096 & 0.095 & 0.052 & 0.124 & 0.116 & 0.090 \\
\hline & Level 2 & 0.010 & 0.023 & 0.036 & 0.068 & 0.065 & 0.032 & 0.081 & 0.075 & 0.061 \\
\hline & Level 3 & 0.011 & 0.018 & 0.035 & 0.070 & 0.070 & 0.034 & 0.087 & 0.084 & 0.058 \\
\hline & Mean & 0.019 & 0.031 & 0.040 & 0.061 & 0.071 & 0.033 & 0.070 & 0.064 & 0.054 \\
\hline & o-value & .00195 & .00195 & .00195 & .00977 & 1.0 & .00195 & & .00586 & .00195 \\
\hline & CLR & & & & & & & & & \\
\hline \multirow[t]{3}{*}{ S1 } & Level 1 & 0.116 & 0.138 & 0.136 & 0.051 & 0.134 & 0.130 & 0.137 & 0.135 & 0.136 \\
\hline & Level 2 & 0.122 & 0.140 & 0.138 & 0.051 & 0.135 & 0.132 & 0.138 & 0.137 & 0.136 \\
\hline & Level 3 & 0.123 & 0.131 & 0.133 & 0.049 & 0.135 & 0.131 & 0.138 & 0.137 & 0.136 \\
\hline \multirow[t]{3}{*}{ S2 } & Level 1 & 0.034 & 0.042 & 0.043 & 0.024 & 0.042 & 0.040 & 0.043 & 0.042 & 0.042 \\
\hline & Level 2 & 0.032 & 0.042 & 0.043 & 0.025 & 0.041 & 0.039 & 0.043 & 0.042 & 0.042 \\
\hline & Level 3 & 0.035 & 0.041 & 0.042 & 0.024 & 0.042 & 0.039 & 0.043 & 0.043 & 0.042 \\
\hline \multirow[t]{3}{*}{ G1 } & Level 1 & 0.062 & 0.136 & 0.147 & 0.047 & 0.129 & 0.112 & 0.147 & 0.138 & 0.145 \\
\hline & Level 2 & 0.067 & 0.135 & 0.145 & 0.046 & 0.126 & 0.106 & 0.142 & 0.126 & 0.138 \\
\hline & Level 3 & 0.065 & 0.111 & 0.132 & 0.046 & 0.119 & 0.104 & 0.135 & 0.124 & 0.134 \\
\hline G2 & Level 1 & 0.042 & 0.081 & 0.095 & 0.026 & 0.090 & 0.078 & 0.105 & 0.100 & 0.104 \\
\hline & Level 2 & 0.041 & 0.078 & 0.091 & 0.026 & 0.083 & 0.072 & 0.097 & 0.095 & 0.095 \\
\hline & Level 3 & 0.042 & 0.066 & 0.084 & 0.026 & 0.081 & 0.069 & 0.094 & 0.091 & 0.093 \\
\hline & Mean & 0.065 & 0.095 & 0.102 & 0.037 & 0.096 & 0.088 & 0.105 & 0.101 & 0.103 \\
\hline & value & .00195 & .00195 & .06446 & .00195 & .00195 & .00195 & & .00195 & .00195 \\
\hline
\end{tabular}


paper, namely MRNET, ARACNE and CLR. Another striking feature is that batch effect removal methods like COMBAT (D3) is able to increase significantly the robustness of network inference algorithms. The results reinforce the idea that normalization alone can not remove batch effects, and therefore the removal of batch effects is essential when merging datasets. In the case of method N, N2 and N3 outperform N1 when MRNET or CLR used. However, in the case of using ARACNE, N1 is as good as N2 while poor results are observed for $\mathrm{N} 3$.

Interestingly, we can clearly observe that $\mathrm{N} 2$ outperforms all three D methods suggesting that assembling networks is better than merging datasets. This could be explained by the fact that gene expression values are very dissimilar in various experiments due to our simulated batch effects (i.e. datasets with different global and local noise). However, the particular combination CLR - D3 offers an exception to this observation. It also should be noted that assembling mutual information matrices ( $M$ methods) surpasses the two other well-known strategies ( $\mathrm{D}$ and $\mathrm{N}$ ) for all datasets under every different levels of distortion, in particular for MRNET (see Figs. 3 and 4) and CLR. Experimental results also show that MRNET benefits the most from meta-analysis and CLR appears to be the most robust. This suggests that while CLR might be a better strategy for analysing individual datasets, MRNET might be a better choice when multiple datasets are available. Although ARACNE appears to be much worse than the two other techniques, that is mainly due to a bad recall (though not visible with AUPR numbers, its precision remains quite competitive). Finally, in the M family of methods, it appears that combining MIM using random effect model (M1) is better than the two other strategies, the internal quality control index (M2) and the median method (M3).

\section{Conclusion}

In the present paper, we proposed a framework for evaluating the different strategies for inferring GRNs from multiple expression datasets. To the best of our knowledge, this is the first systematic evaluation of the two state-of-the-art strategies for the problem of interest, namely "data merging" and "networks ensemble". Furthermore, we presented a new, but promising approach for methods based on coexpression matrices. Indeed, our set of experiments strongly suggest that assembling matrices of pairwise dependencies is a better strategy for network inference than the two commonly used ones. However, there exists many different methods of data and network assembly, as well as experimental conditions that have still to be tested in order to gain a complete understanding of the problem of meta-network inference. Moreover, as mentioned earlier, a

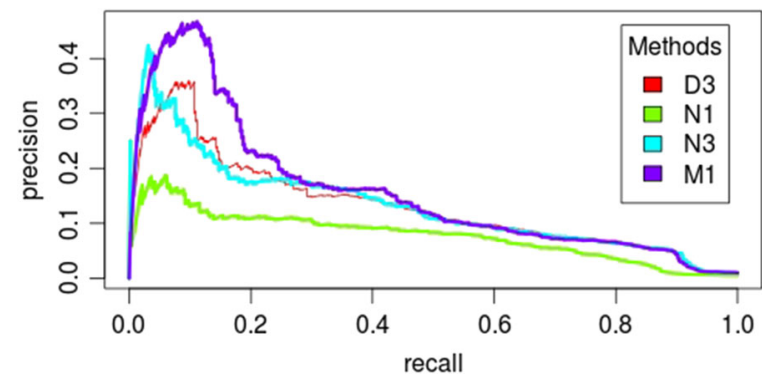

Fig. 3 PR-Curves of method D3, N1, N3 and M1 on dataset S1 at level 1 of data distortion 


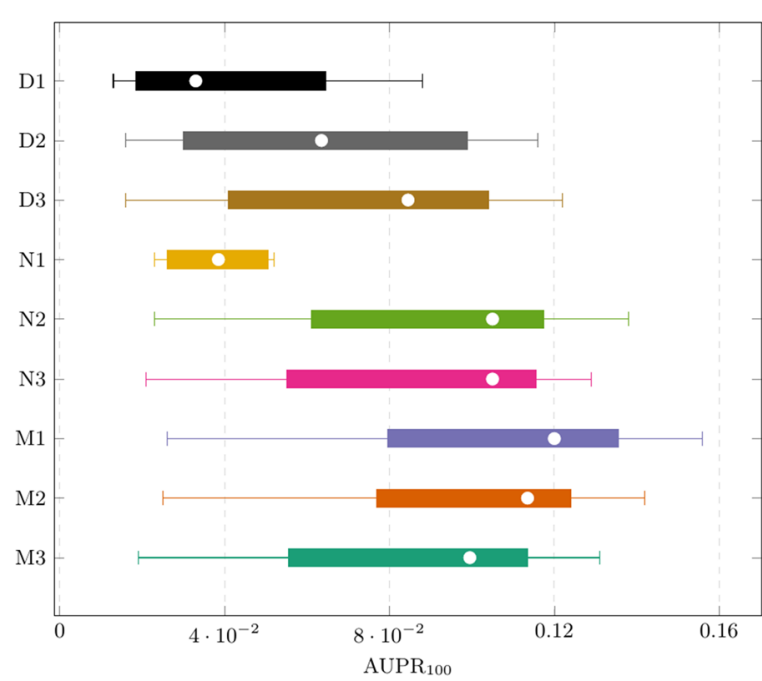

Fig. 4 Boxplots for presented methods using MRNET

large amount of under-exploited transcriptome data of model organisms is now available through public repositories. Thus, additionally to testing new ensemble methods, future works include the use of the best strategy to reconstruct large-scale GRNs of these model organisms.

Abbreviations

AUPR: Area under precision recall; GEO: Gene expression Omnibus; GRNs: Gene regulatory networks; MIM: Mutual information matrix

Acknowledgements

We thank the reviewers from the IEEE DEXA BIOKDD'16 workshop for choosing us as best paper, hereby allowing us to re-publish it here for free.

\section{Funding}

The present research benefited from the use of high performance computing resources ("durandal" grid computer) funded by three grants from the University of Liège: (SFRD-12/03, SFRD-12/04, C-14/73) as well as a Crédit de Recherche of the FNRS under award number nr 23678785. GB was supported by GENGISCAN: GENomic profiling of Gastrointestinal Inflammatory-Sensitive CANcers, [http://mlg.ulb.ac.be/GENGISCAN] Belgian FNRS PDR project.

\section{Availability of data and material}

The datasets analysed during the current study are available from the Netbenchmark Bioconductor package [31].

\section{Authors' contributions}

PEM proposed and supervised the setup of the study. All authors participated in the improvement of the design of the study and the experimental setup. The coding has been implemented by NCP. NCP wrote the first draft of the manuscript. BHK, PB, GB and PEM have revised the manuscript critically for important intellectual content. All authors helped to improve the draft of the manuscript. All authors read and approved the final manuscript.

\section{Competing interests}

The authors declare that they have no competing interests.

\section{Consent for publication}

Not applicable.

Ethics approval and consent to participate

Not applicable.

\section{Publisher's Note}

Springer Nature remains neutral with regard to jurisdictional claims in published maps and institutional affiliations. 


\section{Author details}

${ }^{1}$ Bioinformatics and Systems Biology (BioSys) Lab, Université de Liège, Liège, Belgium. ${ }^{2}$ Princess Margaret Cancer Center, Toronto, ON, Canada. ${ }^{3}$ Department of Medical Biophysics, University of Toronto, Toronto, ON, Canada. ${ }^{4}$ Department of Computer Science, University of Toronto, Toronto, ON, Canada. ${ }^{5}$ Ontario Institute of Cancer Research, Toronto, ON, Canada. ${ }^{6}$ Image Processing group, Technical University of Catalonia, Barcelona, Spain. ${ }^{7}$ Machine Learning Group, Interuniversity Institute of Bioinformatics in Brussels (IB)², Université Libre de Bruxelles, Bruxelles, Belgium.

Received: 3 January 2017 Accepted: 20 April 2017

Published online: 06 May 2017

\section{References}

1. Edgar R, Domrachev M, Lash AE. Gene expression omnibus: Ncbi gene expression and hybridization array data repository. Nucleic Acids Res. 2002;30(1):207-10.

2. Brazma A, Parkinson H, Sarkans U, Shojatalab M, Vilo J, Abeygunawardena N, Holloway E, Kapushesky M, Kemmeren P, Lara GG, et al. Arrayexpress - a public repository for microarray gene expression data at the ebi. Nucleic Acids Res. 2003;31(1):68-71.

3. Marbach D, Costello JC, Küffner R, Vega NM, Prill RJ, Camacho DM, Allison KR, Kellis M, Collins JJ, Stolovitzky G, et al. Wisdom of crowds for robust gene network inference. Nat Methods. 2012;9(8):796-804.

4. Faith JJ, Hayete B, Thaden JT, Mogno I, Wierzbowski J, Cottarel G, Kasif S, Collins JJ, Gardner TS. Large-scale mapping and validation of escherichia coli transcriptional regulation from a compendium of expression profiles. PLOS Biol. 2007;5(1):8.

5. Margolin AA, Nemenman I, Basso K, Wiggins C, Stolovitzky G, Favera RD, Califano A. Aracne: an algorithm for the reconstruction of gene regulatory networks in a mammalian cellular context. BMC Bioinforma. 2006;7(Suppl 1):7.

6. Meyer PE, Kontos K, Bontempi G. Biological network inference using redundancy analysis. In: Bioinformatics Research and Development. Berlin Heidelberg: Springer; 2007. p. 16-27.

7. Meyer PE, Lafitte F, Bontempi G. minet: A r/bioconductor package for inferring large transcriptional networks using mutual information. BMC Bioinforma. 2008;9(1):461.

8. Kugler KG, Mueller LA, Graber A, Dehmer M. Integrative network biology: graph prototyping for co-expression cancer networks. PLoS ONE. 2011;6(7):22843.

9. Taminau J, Meganck S, Lazar C, Steenhoff D, Coletta A, Molter C, Duque R, de Schaetzen V, Solís DYW, Bersini H, et al. Unlocking the potential of publicly available microarray data using insilicodb and insilicomerging r/bioconductor packages. BMC Bioinforma. 2012;13(1):335.

10. Kang DD, Sibille E, Kaminski N, Tseng GC. Metaqc: objective quality control and inclusion/exclusion criteria for genomic meta-analysis. Nucleic Acids Res. 2012;40(2):15.

11. Ramasamy A, Mondry A, Holmes CC, Altman DG. Key issues in conducting a meta-analysis of gene expression microarray datasets. PLoS Med. 2008;5(9):184.

12. Wirapati $P$, Sotiriou $C$, Kunkel $S$, Farmer $P$, Pradervand $S$, Haibe-Kains $B$, Desmedt $C$, Ignatiadis $M$, Sengstag $T$, Schutz F, et al. Meta-analysis of gene expression profiles in breast cancer: toward a unified understanding of breast cancer subtyping and prognosis signatures. Breast Cancer Res. 2008;10(4):65.

13. Desmedt C, Haibe-Kains B, Wirapati P, Buyse M, Larsimont D, Bontempi G, Delorenzi M, Piccart M, Sotiriou C. Biological processes associated with breast cancer clinical outcome depend on the molecular subtypes. Clin Cancer Res. 2008;14(16):5158-165.

14. Hong F, Breitling R, McEntee CW, Wittner BS, Nemhauser JL, Chory J. Rankprod: a bioconductor package for detecting differentially expressed genes in meta-analysis. Bioinformatics. 2006;22(22):2825-827.

15. Huber W, Von Heydebreck A, Sültmann H, Poustka A, Vingron M. Variance stabilization applied to microarray data calibration and to the quantification of differential expression. Bioinformatics. 2002;18(suppl 1):96-104.

16. Belcastro V, Siciliano V, Gregoretti F, Mithbaokar P, Dharmalingam G, Berlingieri S, lorio F, Oliva G, Polishchuck R, Brunetti-Pierri N, et al. Transcriptional gene network inference from a massive dataset elucidates transcriptome organization and gene function. Nucleic Acids Res. 2011;39(20):8677-688.

17. Adler P, Kolde R, Kull M, Tkachenko A, Peterson H, Reimand J, Vilo J. Mining for coexpression across hundreds of datasets using novel rank aggregation and visualization methods. Genome Biol. 2009;10(12):139.

18. Leek JT, Scharpf RB, Bravo HC, Simcha D, Langmead B, Johnson WE, Geman D, Baggerly K, Irizarry RA. Tackling the widespread and critical impact of batch effects in high-throughput data. Nat Rev Genet. 2010;11(10):733-9.

19. Irizarry RA, Hobbs B, Collin F, Beazer-Barclay YD, Antonellis KJ, Scherf U, Speed TP. Exploration, normalization, and summaries of high density oligonucleotide array probe level data. Biostatistics. 2003;4(2):249-64.

20. Dyrskjøt L, Kruhøffer M, Thykjaer T, Marcussen N, Jensen JL, Møller K, Ørntoft TF. Gene expression in the urinary bladder a common carcinoma in situ gene expression signature exists disregarding histopathological classification. Cancer Res. 2004;64(11):4040-048.

21. Johnson WE, Li C, Rabinovic A. Adjusting batch effects in microarray expression data using empirical bayes methods. Biostatistics. 2007;8(1):118-27.

22. Hase T, Ghosh S, Yamanaka R, Kitano H. Harnessing diversity towards the reconstructing of large scale gene regulatory networks. PLoS Comput Biol. 2013;9(11):1003361.

23. Ding C, Peng H. Minimum redundancy feature selection from microarray gene expression data. J Bioinforma Comput Biol. 2005;3(02):185-205.

24. Sims AH, Smethurst GJ, Hey Y, Okoniewski MJ, Pepper SD, Howell A, Miller CJ, Clarke RB. The removal of multiplicative, systematic bias allows integration of breast cancer gene expression datasets-improving meta-analysis and prediction of prognosis. BMC Med Genet. 2008;1(1):1.

25. Cheadle C, Vawter MP, Freed WJ, Becker KG. Analysis of microarray data using z score transformation. J Mol Diagn. 2003;5(2):73-81.

26. Chen C, Grennan K, Badner J, Zhang D, Gershon E, Jin L, Liu C. Removing batch effects in analysis of expression microarray data: an evaluation of six batch adjustment methods. PloS ONE. 2011;6(2):17238. 
27. Lazar C, Meganck S, Taminau J, Steenhoff D, Coletta A, Molter C, Weiss-Solís DY, Duque R, Bersini H, Nowé A. Batch effect removal methods for microarray gene expression data integration: a survey. Brief Bioinform. 2012037.

28. Bellot Pujalte P, Salembier Clairon PJ, Oliveras Vergés A, Meyer P. Study of normalization and aggregation approaches for consensus network estimation. In: IEEE SSCI 2015: 2015 IEEE Symposium Series on Computational Intelligence; 7-10 December 2015, Cape Town, South Afrika. Institute of Electrical and Electronics Engineers (IEEE); 2015. p. 1-6.

29. Wang K, Narayanan M, Zhong H, Tompa M, Schadt EE, Zhu J. Meta-analysis of inter-species liver co-expression networks elucidates traits associated with common human diseases. PLoS Comput Biol. 2009;5(12):1000616.

30. Schmidt FL, Hunter JE. Methods of Meta-analysis: Correcting Error and Bias in Research Findings. Los Angeles: Sage publications; 2014.

31. Bellot $P$, Olsen C, Salembier P, Oliveras-Vergés A, Meyer PE. Netbenchmark: a bioconductor package for reproducible benchmarks of gene regulatory network inference. BMC Bioinforma. 2015;16(1):1.

32. Emmert-Streib F, Dehmer M, Haibe-Kains B. Gene regulatory networks and their applications: understanding biological and medical problems in terms of networks. Front Cell Dev Biol. 2014;2:38.

33. Davis J, Goadrich M. The relationship between precision-recall and roc curves. In: Proceedings of the 23rd International Conference on Machine Learning. ACM; 2006. p. 233-40.

34. Cuzick J. A wilcoxon-type test for trend. Stat Med. 1985;4(4):543-7.

35. Benjamini Y, Hochberg Y. Controlling the false discovery rate: a practical and powerful approach to multiple testing J R Stat Soc Ser B Methodol. 1995:289-300.

Submit your next manuscript to BioMed Central and we will help you at every step:

- We accept pre-submission inquiries

- Our selector tool helps you to find the most relevant journal

- We provide round the clock customer support

- Convenient online submission

- Thorough peer review

- Inclusion in PubMed and all major indexing services

- Maximum visibility for your research 